\title{
Existence and Multiplicity of Periodic Solutions for Nonautonomous Second-Order Discrete Hamiltonian Systems
}

\author{
CHUNGEN LIU* AND YuYOU ZHONG
}

ABSTRACT. In this paper, we consider the periodic solutions of the following non-autonomous second order discrete system

$$
\Delta^{2} u(n-1)=\nabla F(n, u(n)), \quad n \in \mathbb{Z} .
$$

When the nonlinear function $F(n, x)$ is like-quadratic for $x$, we obtain some existence and multiplicity results under twisting conditions by using the least action principle and a multiple critical point theorem.

Keywords: Periodic solution, second-order discrete Hamiltonian system, the least action principle, critical point theory.

2020 Mathematics Subject Classification: 35R02, 58J05.

\section{INTRODUCTION AND MAIN RESULTS}

In this paper, we consider the periodic solutions of the following nonautonomous second order discrete Hamiltonian system

$$
\Delta^{2} u(n-1)=\nabla F(n, u(n)), u(n) \in \mathbb{R}^{N}, n \in \mathbb{Z},
$$

where $\Delta u(n)=u(n+1)-u(n), \Delta^{2} u(n)=\Delta(\Delta u(n))$ and $\nabla F(n, x)$ denotes the gradient of the function $F$ with respect to the second variable $x$. $F$ satisfies the following condition:

$$
\begin{aligned}
& F(n, \cdot) \in C^{1}\left(\mathbb{R}^{N}, \mathbb{R}\right), \forall n \in \mathbb{Z} ; \\
& F(n+T, x)=F(n, x), \forall(n, x) \in \mathbb{Z} \times \mathbb{R}^{N}, T \in \mathbb{Z} \text { and } T \geq 2 .
\end{aligned}
$$

Historically, in 2003, Guo and Yu, first considered the existence of periodic solutions of difference equations as (1.1) via variational method and critical point theory in three papers $[2,3,4]$. In 2004, Zhou, Yu and Guo [11], further studied the existence and multiplicity of periodic solutions of the discrete Hamiltonian system (1.1). After that, the existence and multiplicity of periodic solutions for system (1.1) have been extensively studied and many interesting results were obtained. We refer the readers to $[5,6,8,9,10]$ and the references therein for these topics. Among them, we should mention some work which have relation with our work of this paper. For the condition on $F$, Guo and Yu in [3], first required the nonlinearity $\nabla F(n, x)$ is sub-linear included the bounded case. We say that the nonlinearity $\nabla F(n, x)$ is growing sublinearly if there exist $M_{1}>0, M_{2}>0$ and $\alpha \in[0,1)$ such that

$$
|\nabla F(t, x)| \leq M_{1}|x|^{\alpha}+M_{2}, \quad \forall(n, x) \in \mathbb{Z}[1, T] \times \mathbb{R}^{N},
$$


where $\mathbb{Z}[a, b]:=\mathbb{Z} \cap[a, b]$ for all $a, b \in \mathbb{Z}$ with $a \leq b$. Xue and Tang in [9] used the least action principle to verified that system (1.1) possesses at least one $T$-periodic solution with the assumption of

$$
\lim _{|x| \rightarrow+\infty}|x|^{-2 \alpha} \sum_{n=1}^{T} F(n, x)=+\infty .
$$

We also refer the paper [3] for this topic. In the case of $\alpha=1$, assumption (1.2) becomes to the following assumption in which the nonlinearity $\nabla F(n, x)$ does not exceed linear growth, that is, there are $M_{3}>0$ and $M_{4} \geq 0$, such that

$$
|\nabla F(n, x)| \leq M_{3}|x|+M_{4}, \quad \forall(n, x) \in \mathbb{Z}[1, T] \times \mathbb{R}^{N} .
$$

The case $\nabla F(n, x)=A x+B$ satisfies the condition (1.4). It is well known that in this case the system (1.1) in general does not possess a solution. A twisting condition is required to avoid this case. Considering the nonlinearity $\nabla F(n, x)$ which is the sum of assumption (1.2) and (1.4), $\mathrm{Hu}$ [6] also used the least action principle to verify that system (1.1) possesses at least one $T$-periodic solution under a twisting condition which is included in the following case

$$
\lim _{|x| \rightarrow+\infty}|x|^{-2} \sum_{n=1}^{T} F(n, x)>-\infty .
$$

When the nonlinearity $\nabla F(n, x)$ meets the following assumption that there are $f, g: \mathbb{Z}[1, T] \rightarrow$ $\mathbb{R}^{+}$and $\alpha \in(0,1)$, such that

$$
|\nabla F(t, x)| \leq f(n)|x|^{\alpha}+g(n), \quad \forall(n, x) \in \mathbb{Z}[1, T] \times \mathbb{R}^{N}
$$

or

$$
|\nabla F(t, x)| \leq f(n)|x|+g(n), \quad \forall(n, x) \in \mathbb{Z}[1, T] \times \mathbb{R}^{N} .
$$

Tang and Zhang [8] obtained some existence results for the $T$-periodic solutions of system (1.1) under some different twisting conditions.

In this paper, we will further study the existence and multiplicity of $T$-periodic solutions of (1.5) with some different twisting conditions. The following are our main results.

Theorem 1.1. Suppose that $F(n, x)=F_{1}(n, x)+F_{2}(n, x)$ with $F_{1}$ and $F_{2}$ satisfying the conditions $(A)$ and the following three growing conditions:

$\left(B_{1}\right)$ There exist $f, g: \mathbb{Z}[1, T] \rightarrow \mathbb{R}^{+}$and $\alpha \in[0,1)$, such that

$$
\left|\nabla F_{1}(n, x)\right| \leq f(n)|x|^{\alpha}+g(n) .
$$

$\left(B_{2}\right) F_{2}(n, x)$ satisfies condition (1.4), i.e., there exist constants $M_{3}, M_{4} \in \mathbb{R}^{+}$such that

$$
\left|\nabla F_{2}(n, x)\right| \leq M_{3}|x|+M_{4}, M_{3}<\lambda_{1}:=\lambda_{1}=2-2 \cos \frac{2 \pi}{T} .
$$

$\left(B_{3}\right) F$ satisfies that

$$
\lim _{|x| \rightarrow+\infty} \inf |x|^{-2} \sum_{n=1}^{T} F(n, x)>\frac{M_{3}^{2} T}{2\left(\lambda_{1}-M_{3}\right)} .
$$

Then, system (1.1) possesses at least one T-periodic solution that minimizes the functional $\varphi$ given by

$$
\varphi(u)=\frac{1}{2} \sum_{n=1}^{T}|\Delta u(n)|^{2}+\sum_{n=1}^{T} F(n, u(n))
$$


in the Hilbert space $H_{T}$ defined by

$$
H_{T}=\left\{u: \mathbb{Z} \rightarrow \mathbb{R}^{N} \mid u(n+T)=u(n), n \in \mathbb{Z}\right\} .
$$

Remark 1.1. The condition $\left(B_{3}\right)$ is twisted with the conditions $\left(B_{1}\right)$ and $\left(B_{2}\right)$. Our condition $\left(B_{3}\right)$ in Theorem 1.1 is different form the condition $\left(A_{4}\right)$ in Theorem 1 of [6]. In Theorem 1.1, when $F_{1}(n, x) \equiv$ 0, comparing with Theorem 1.3 of [8], the condition $\left(B_{3}\right)$ in some sense is loose for some choices of $T$, one can check it for $T=2,3,4,5,6$ and so on.

Theorem 1.2. Suppose $F(n, x)=F_{1}(n, x)+F_{2}(n, x)$ satisfying condition $(A)$ with $F_{1}$ and $F_{2}$ satisfying the following three growing conditions:

$\left(B_{4}\right) F_{1}$ satisfies the condition (1.7), i.e., there exist $f, g: \mathbb{Z}[1, T] \rightarrow \mathbb{R}^{+}$such that

$$
\left|\nabla F_{1}(n, x)\right| \leq f(n)|x|+g(n), \sum_{n=1}^{T} f(n)<\lambda_{1} .
$$

( $\left.B_{5}\right) F_{2}$ satisfies the condition (1.2), i.e., there are some constants $M_{1}, M_{2} \in \mathbb{R}^{+}$and $\alpha \in[0,1)$ such that

$$
\left|\nabla F_{2}(n, x)\right| \leq M_{1}|x|^{\alpha}+M_{2}
$$

$\left(B_{6}\right) F$ satisfies that

$$
\lim _{|x| \rightarrow+\infty} \inf |x|^{-2} \sum_{n=1}^{T} F(n, x)>\frac{1}{2\left(\lambda_{1}-\sum_{n=1}^{T} f(n)\right)}\left(\sum_{n=1}^{T} f(n)\right)^{2} .
$$

Then, system (1.1) possesses at least one T-periodic solution.

Remark 1.2. Conditions of $\left(B_{4}\right)$ and $\left(B_{6}\right)$ in Theorem 1.2 are different form conditions of $\left(A_{3}\right)$ and $\left(A_{4}\right)$ in Theorem 1 of [6], respectively. Condition $\left(B_{6}\right)$ in Theorem 1.2 is different form condition of (1.14) in Theorem 1.3 of [8].

Theorem 1.3. Suppose that $F(n, x)$ satisfies $(A),\left(B_{1}\right),\left(B_{2}\right),\left(B_{3}\right)$ and $\left(A_{1}\right)$ there are some constants $\delta>0, k \in \mathbb{Z}\left[0,\left[\frac{T}{2}-1\right]\right]$ such that

$$
-\frac{1}{2} \lambda_{k+1}|x|^{2} \leq F(n, x) \leq-\frac{1}{2} \lambda_{k}|x|^{2},
$$

$\forall x \in \mathbb{R}^{N}$ with $|x|<\delta$ and $\forall n \in[1, T]$, where $\lambda_{k}=2-2 \cos k \omega, \omega=\frac{2 \pi}{T}, T>2,[a]=\max \{k \in$ $\mathbb{Z} \mid k \leq a\}$ denotes the Gauss Function. Then, system (1.1) has at least two T-periodic solutions.

Parallelly, we have the following result.

Theorem 1.4. Suppose that $F(n, x)$ satisfies $(A),\left(B_{4}\right),\left(B_{5}\right),\left(B_{6}\right)$ and $\left(A_{1}\right)$. Then, system (1.1) has at least two T-periodic solutions.

\section{SOME IMPORTANT LEMMAS}

$H_{T}:=\left\{u: \mathbb{Z} \rightarrow \mathbb{R}^{N} \mid u(n+T)=u(n), n \in \mathbb{Z}\right\}$ can be equipped with the inner product

$$
\langle u, v\rangle=\sum_{n=1}^{T}(u(n), v(n)), \quad \forall u, v \in H_{T},
$$

so the norm $\|\cdot\|$ is

$$
\|u\|=\left(\sum_{n=1}^{T}|u(n)|^{2}\right)^{\frac{1}{2}}, \quad \forall u \in H_{T}
$$


where $(\cdot, \cdot)$ and $|$.$| denotes the usual inner product and the usual norm in \mathbb{R}^{N}$, respectively. It is easy to verify that $\left(H_{T},\langle\cdot, \cdot\rangle\right)$ is a finite dimensional Hilbert space and linear homeomorphic to $\mathbb{R}^{N T}$.

For every positive number $r>1$, we can equip $H_{T}$ with another norm $\|u\|_{r}$, where

$$
\|u\|_{r}=\left(\sum_{n=1}^{T}|u(n)|^{r}\right)^{\frac{1}{r}}, \quad \forall u \in H_{T} .
$$

Distinctly, $\|u\|_{2}=\|u\|$ and $\left(H_{T},\|u\|_{2}\right)$ is equivalent to $\left(H_{T},\|u\|_{r}\right)$ for $r>1$. Thus, there are two constants $C_{2} \geq C_{1}>0$, such that $\forall u \in H_{T}$

$$
C_{1}\|u\|_{r} \leq\|u\| \leq C_{2}\|u\|_{r} .
$$

For system (1.1), Xue and Tang [10] verify that the problem of seeking $T$-periodic solutions is equal to that of finding the critical points of $\varphi(u)$ defined in (1.8) on $H_{T}$.

To prove our results, we now give four useful lemmas.

Lemma 2.1. ([10]) As a subspace of $H_{T}, N_{k}$ is defined by:

$$
N_{k}:=\left\{u \in H_{T} \mid-\Delta^{2} u(n-1)=\lambda_{k} u(n)\right\},
$$

where $\lambda_{k}=2-2 \cos k \omega, \omega=\frac{2 \pi}{T}, k \in \mathbb{Z}\left[0,\left[\frac{T}{2}\right]\right]$. The following statements hold:

(i) $N_{k} \perp N_{j}, k \neq j, k, j \in \mathbb{Z}\left[0,\left[\frac{T}{2}\right]\right]$,

(ii) $H_{T}=\bigoplus_{k=0}^{\left[\frac{T}{2}\right]} N_{k}$.

Lemma 2.2 ([10]). Define $H_{k}:=\bigoplus_{j=0}^{k} N_{j}, H_{k}^{\perp}:=\bigoplus_{j=k+1}^{[T / 2]} N_{j}, k \in \mathbb{Z}[0,[T / 2]-1]$, then one has

$$
\begin{aligned}
& \sum_{n=1}^{T}|\Delta u(n)|^{2} \leq \lambda_{k}\|u\|^{2}, \quad \forall u \in H_{k} ; \\
& \sum_{n=1}^{T}|\Delta u(n)|^{2} \geq \lambda_{k+1}\|u\|^{2}, \quad \forall u \in H_{k}^{\perp} .
\end{aligned}
$$

Lemma 2.3 ([7]). If $\varphi$ is weakly lower semi continuous on a reflexive Banach space $X$ and has a bounded minimizing sequence, then $\varphi$ has a minimum on $X$.

Lemma 2.4 ([1]). Let $\varphi$ be a $C^{1}$ function on $X=X_{1} \bigoplus X_{2}$ with $\varphi(0)=0$, satisfying $(P S)$ condition and for some $\delta>0$,

$$
\begin{aligned}
& \varphi(u) \geq 0 \quad \text { for } u \in X_{1},\|u\| \leq \delta, \\
& \varphi(u) \leq 0 \quad \text { for } u \in X_{2},\|u\| \leq \delta .
\end{aligned}
$$

Assume also that $\varphi$ is bounded below and $\inf _{X} \varphi<0$, then $\varphi$ has at least two nonzero critical points.

By Lemma 2.1, one rewrites $u$ as

$$
u=\bar{u}+\tilde{u} \in N_{0} \bigoplus N_{0}^{\perp},
$$

where $\bar{u}=(1 / T) \sum_{n=1}^{T} u(n)$. 
By (2.9), (2.10) and (2.12), one has

$$
\begin{aligned}
\|u\| & =\left(\sum_{n=1}^{T}|u(n)|^{2}\right)^{\frac{1}{2}}=\left(\sum_{n=1}^{T}|\bar{u}+\tilde{u}(n)|^{2}\right)^{\frac{1}{2}} \\
& =\left(\sum_{n=1}^{T}(\bar{u}+\tilde{u}(n), \bar{u}+\tilde{u}(n))\right)^{\frac{1}{2}} \\
& =\left(\sum_{n=1}^{T}\left(|\bar{u}|^{2}+|\tilde{u}(n)|^{2}\right)\right)^{\frac{1}{2}}=\left(T|\bar{u}|^{2}+\|\tilde{u}\|^{2}\right)^{\frac{1}{2}} .
\end{aligned}
$$

Then, one has

$$
\|u\| \leq \sqrt{T+1}\left(|\bar{u}|^{2}+\|\tilde{u}\|^{2}\right)^{\frac{1}{2}} \quad \text { and } \quad\|u\| \geq\left(|\bar{u}|^{2}+\|\tilde{u}\|^{2}\right)^{\frac{1}{2}} .
$$

Therefore, one has that $\|u\| \rightarrow \infty$ if and only if $\left(|\bar{u}|+\|\tilde{u}\|^{2}\right)^{\frac{1}{2}} \rightarrow \infty$.

\section{PROOF OF MAIN RESULTS}

Since the proof of Theorem 1.4 is similar to that of Theorem 1.3, we only prove Theorem 1.1, Theorem 1.2 and Theorem 1.3 in this section.

For convenience, we denote

$$
R_{1}=\sum_{n=1}^{T} f(n), \quad R_{2}=\sum_{n=1}^{T} g(n) .
$$

Proof of Theorem 1.1. According to $\left(B_{3}\right)$, we can choose a positive constant $a_{1}$, such that

$$
a_{1}>\frac{\varepsilon+M_{3}}{\lambda_{1}-M_{3}}>0
$$

for a small number $\varepsilon>0$ and

$$
\lim _{|x| \rightarrow+\infty} \inf |x|^{-2} \sum_{n=1}^{T} F(n, x)>\frac{a_{1}}{2} M_{3} T .
$$

By $\left(B_{1}\right)$, we obtain

$$
\begin{aligned}
& \left|\sum_{n=1}^{T}\left[F_{1}(n, u(n))-F_{1}(n, \bar{u})\right]\right| \\
= & \left|\sum_{n=1}^{T} \int_{0}^{1}\left(\nabla F_{1}(n, \bar{u}+s \tilde{u}(n)), \tilde{u}(n)\right) d s\right| \\
\leq & \sum_{n=1}^{T} \int_{0}^{1} f(n)|\bar{u}+s \tilde{u}(n)|^{\alpha}|\tilde{u}(n)| d s+\sum_{n=1}^{T} \int_{0}^{1} g(n)|\tilde{u}(n)| d s \\
\leq & \sum_{n=1}^{T} f(n)\left(|\bar{u}|^{\alpha}+|\tilde{u}(n)|^{\alpha}\right)|\tilde{u}(n)|+\sum_{n=1}^{T} g(n)|\tilde{u}(n)|
\end{aligned}
$$




$$
\begin{aligned}
& \leq R_{1}|\bar{u}|^{\alpha}\|\tilde{u}\|_{\infty}+R_{1}\|\tilde{u}\|_{\infty}^{\alpha+1}+R_{2}\|\tilde{u}\|_{\infty} \\
& \leq \frac{\varepsilon}{2 a_{1}}\|\tilde{u}\|_{\infty}^{2}+\frac{a_{1}}{2 \varepsilon} R_{1}^{2}|\bar{u}|^{2 \alpha}+R_{1}\|\tilde{u}\|_{\infty}^{\alpha+1}+R_{2}\|\tilde{u}\|_{\infty} \\
& \leq \frac{\varepsilon}{2 a_{1}}\|\tilde{u}\|^{2}+\frac{a_{1}}{2 \varepsilon} R_{1}^{2}|\bar{u}|^{2 \alpha}+R_{1}\|\tilde{u}\|^{\alpha+1}+R_{2}\|\tilde{u}\|
\end{aligned}
$$

for any $u \in H_{T}$ with $\|u\|_{\infty}:=\max _{n \in \mathbb{Z} \cap[1, T]}|u(n)|$.

By $\left(B_{2}\right)$, we have

$$
\begin{aligned}
& \left|\sum_{n=1}^{T}\left[F_{2}(u(n))-F_{2}(\bar{u})\right]\right| \\
= & \left|\sum_{n=1}^{T} \int_{0}^{1}\left(\nabla F_{2}(\bar{u}+s \tilde{u}(n)), \tilde{u}(n)\right) d s\right| \\
\leq & \sum_{n=1}^{T} \int_{0}^{1} M_{3}(|\bar{u}+s \tilde{u}(n)|)|\tilde{u}(n)| d s+\sum_{n=1}^{T} \int_{0}^{1} M_{4}|\tilde{u}(n)| d s \\
\leq & M_{3} \sum_{n=1}^{T}\left(|\bar{u}|+\frac{1}{2}|\tilde{u}(n)|\right)|\tilde{u}(n)|+\sum_{n=1}^{T} M_{4}|\tilde{u}(n)| \\
\leq & M_{3} \sum_{n=1}^{T}|\bar{u}||\tilde{u}(n)|+\frac{M_{3}}{2} \sum_{n=1}^{T}|\tilde{u}|^{2}+M_{4} \sum_{n=1}^{T}|\tilde{u}| \\
\leq & M_{3}\left(\sum_{n=1}^{T}|\bar{u}|^{2}\right)^{\frac{1}{2}}\left(\sum_{n=1}^{T}|\tilde{u}(n)|^{2}\right)^{\frac{1}{2}}+\frac{M_{3}}{2} \sum_{n=1}^{T}|\tilde{u}|^{2}+M_{4} \sum_{n=1}^{T}|\tilde{u}| \\
\leq & \frac{a_{1}}{2} M_{3} \sum_{n=1}^{T}|\bar{u}|^{2}+\frac{M_{3}}{2 a_{1}} \sum_{n=1}^{T}|\tilde{u}|^{2}+\frac{M_{3}}{2} \sum_{n=1}^{T}|\tilde{u}|^{2}+M_{4} \sum_{n=1}^{T}|\tilde{u}| \\
\leq & \frac{a_{1}}{2} M_{3} T|\bar{u}|^{2}+\left(\frac{M_{3}}{2 a_{1}}+\frac{M_{3}}{2}\right)\|\tilde{u}\|^{2}+M_{4} \sqrt{T}\|\tilde{u}\|
\end{aligned}
$$

for any $u \in H_{T}$.

Hence, by (1.8), (3.15), (3.16) and Lemma 2.2, we have

$$
\begin{aligned}
\varphi(u) & \geq\left(\frac{\lambda_{1}}{2}-\frac{\varepsilon}{2 a_{1}}-\frac{M_{3}}{2 a_{1}}-\frac{M_{3}}{2}\right)\|\tilde{u}\|^{2}-\left(R_{2}+M_{4} \sqrt{T}\right)\|\tilde{u}\|-R_{1}\|\tilde{u}\|^{\alpha+1} \\
& +|\bar{u}|^{2}\left(|\bar{u}|^{-2} \sum_{n=1}^{T} F(n, \bar{u})-\frac{a_{1}}{2} M_{3} T\right)-\frac{a_{1}}{2 \varepsilon} R_{1}^{2}|\bar{u}|^{2 \alpha} .
\end{aligned}
$$

Since $u=\bar{u}+\tilde{u} \in N_{0} \bigoplus N_{0}^{\perp}$, (3.13), (3.14) and (3.17) imply that

$$
\varphi(u) \rightarrow+\infty, \quad\|u\| \rightarrow \infty .
$$

Thus, $\varphi$ is coercive. Since $\varphi$ is continuous, it possesses a bounded minimizing sequence in the finite dimensional Hilbert space $H_{T}$. Therefore, by Lemma 2.3, we obtain a critical point $u$ which is a $T$-periodic solution of system (1.1) and the minimizer of the function $\varphi$. The proof is complete.

By (3.15) in which the number $\frac{\varepsilon}{a_{1}}$ should be replaced by $\lambda_{1}-\varepsilon$ and $M_{3}=0$ in (3.16), we have the following result. 
Theorem 3.5. Suppose that $F(n, x)$ with $F_{2}=0$ satisfying $(A),\left(B_{1}\right)$ and $\left(B_{3}^{\prime}\right) \quad \lim _{|x| \rightarrow+\infty} \inf |x|^{-2 \alpha} \sum_{n=1}^{T} F(n, x)>\frac{M_{1}^{2}}{2 \lambda_{1}}$.

Then, system (1.1) has at least one T-periodic solution that minimizes the functional $\varphi$ in the Hilbert space $H_{T}$.

Comparing with Theorem 1.1 of [8], we see that the condition $\left(B_{3}^{\prime}\right)$ is loose for some choices of $T$, for example $T=2,3,4,5$ and so on. In Theorem 1.1, when $F_{1}(n, x) \equiv 0$, comparing with Theorem 1.3 of [8], the condition $\left(B_{3}\right)$ in some sense is loose for some choices of $T$, one can check it for $T=2,3,4,5,6$ and so on.

Now, we give a proof of Theorem 1.2.

Proof of Theorem 1.2. By $\left(B_{6}\right)$, we can choose a positive constant $a_{2}$ and $\varepsilon>0$, such that

$$
a_{2}>\frac{1}{\lambda_{1}-R_{1}-\varepsilon}
$$

and

$$
\lim _{|x| \rightarrow \infty} \inf |x|^{-2} \sum_{n=1}^{T} F(n)>\frac{a_{2}}{2} R_{1}^{2} .
$$

By $\left(B_{4}\right), \forall u \in H_{T}$, we have

$$
\begin{aligned}
& \left|\sum_{n=1}^{T}\left[F_{1}(n, u(n))-F_{1}(n, \bar{u})\right]\right| \\
= & \left|\sum_{n=1}^{T} \int_{0}^{1}\left(\nabla F_{1}(n, \bar{u}+s \tilde{u}(n)), \tilde{u}(n)\right) d s\right| \\
\leq & \sum_{n=1}^{T} \int_{0}^{1} f(n)|\bar{u}+s \tilde{u}(n)||\tilde{u}(n)| d s+\sum_{n=1}^{T} \int_{0}^{1} g(n)|\tilde{u}(n)| d s \\
\leq & \sum_{n=1}^{T} f(n)\left(|\bar{u}|+\frac{1}{2}|\tilde{u}(n)|\right)|\tilde{u}(n)|+\sum_{n=1}^{T} g(n)|\tilde{u}(n)| \\
\leq & R_{1}|\bar{u}|\|\tilde{u}\|_{\infty}+\frac{R_{1}}{2}\|\tilde{u}\|_{\infty}^{2}+R_{2}\|\tilde{u}\|_{\infty} \\
\leq & \frac{1}{2 a_{2}}\|\tilde{u}\|_{\infty}^{2}+\frac{a_{2}}{2} R_{1}^{2}|\bar{u}|^{2}+\frac{R_{1}}{2}\|\tilde{u}\|_{\infty}^{2}+R_{2}\|\tilde{u}\|_{\infty} \\
= & \left(\frac{1}{2 a_{2}}+\frac{R_{1}}{2}\right)\|\tilde{u}\|_{\infty}^{2}+R_{2}\|\tilde{u}\|_{\infty}+\frac{a_{2}}{2} R_{1}^{2}|\bar{u}|^{2} \\
\leq & \left(\frac{1}{2 a_{2}}+\frac{R_{1}}{2}\right)\|\tilde{u}\|^{2}+R_{2}\|\tilde{u}\|+\frac{a_{2}}{2} R_{1}^{2}|\bar{u}|^{2} .
\end{aligned}
$$

By $\left(B_{5}\right)$, we have

$$
\begin{aligned}
& \left|\sum_{n=1}^{T}\left[F_{2}(n, u(n))-F_{2}(n, \bar{u})\right]\right| \\
= & \left|\sum_{n=1}^{T} \int_{0}^{1}\left(\nabla F_{2}(n, \bar{u}+s \tilde{u}(n)), \tilde{u}(n)\right) d s\right|
\end{aligned}
$$




$$
\begin{aligned}
& \leq \sum_{n=1}^{T} \int_{0}^{1} M_{1}|\bar{u}+s \tilde{u}(n)|^{\alpha}|\tilde{u}(n)| d s+\sum_{n=1}^{T} \int_{0}^{1} M_{2}|\tilde{u}(n)| d s \\
& \leq \sum_{n=1}^{T} M_{1}\left(|\bar{u}|^{\alpha}+|\tilde{u}(n)|^{\alpha}\right)|\tilde{u}(n)|+\sum_{n=1}^{T} M_{2}|\tilde{u}(n)| \\
& \leq M_{1} \sqrt{T}|\bar{u}|^{\alpha}\|\tilde{u}\|+M_{1} \sum_{n=1}^{T}|\tilde{u}|^{\alpha+1}+M_{2} \sum_{n=1}^{T}|\tilde{u}(n)| \\
& \leq \frac{T M_{1}^{2}}{2 \varepsilon}|\bar{u}|^{2 \alpha}+\frac{\varepsilon}{2}\|\tilde{u}\|^{2}+C_{1}\|\tilde{u}\|^{\alpha+1}+C_{2}\|\tilde{u}\| .
\end{aligned}
$$

Hence, by (1.8), (3.20), (3.21) and Lemma 2.2, we have

$$
\begin{aligned}
\varphi(u) & \geq\left(\frac{\lambda_{1}}{2}-\frac{1}{2 a_{2}}-\frac{R_{1}}{2}-\frac{\varepsilon}{2}\right)\|\tilde{u}\|^{2}-C_{1}\|\tilde{u}\|^{\alpha+1}-\left(C_{2}+R_{2}\right)\|\tilde{u}(n)\| \\
& +|\bar{u}|^{2}\left(|\bar{u}|^{-2} \sum_{n=1}^{T} F(n, \bar{u})-\frac{a_{2}}{2} R_{1}^{2}\right)-\frac{T M_{1}^{2}}{2 \varepsilon}|\bar{u}|^{2 \alpha} .
\end{aligned}
$$

Since $u=\bar{u}+\tilde{u} \in N_{0} \oplus N_{0}^{\perp}$, (3.18), (3.19) and (3.22) imply that

$$
\varphi(u) \rightarrow+\infty, \quad\|u\| \rightarrow \infty,
$$

that is, $\varphi$ is coercive. It is easy to verify that there exists a bounded minimizing sequence which insures that $\varphi$ possesses a minimal point in the finite dimensional Hilbert space $H_{T}$ by Lemma 2.3. The proof is complete.

Proof of Theorem 1.3. According to the proof of Theorem 1.1, we can implies that $\varphi$ is bounded below and satisfies the $(P S)$ condition. By $\left(A_{1}\right)$ and Lemma 2.2, one has

$$
\varphi(u) \leq \frac{1}{2} \lambda_{k}\|u\|^{2}+\sum_{n=1}^{T}\left(-\frac{1}{2} \lambda_{k}|u|^{2}\right)=0
$$

for any $u \in H_{k}$ with $\|u\| \leq \delta$ and

$$
\varphi(u) \geq \frac{1}{2} \lambda_{k+1}\|u\|^{2}+\sum_{n=1}^{T}\left(-\frac{1}{2} \lambda_{k+1}|u|^{2}\right)=0
$$

for any $u \in H_{k}^{\perp}$ with $\|u\| \leq \delta$.

If $\inf _{u \in H_{T}} \varphi(u)<0$, we completed our proof of Theorem 1.3 by Lemma 2.4.

If $\inf _{u \in H_{T}} \varphi(u) \geq 0$, by (3.23) and (3.24), we have $\varphi(u)=\inf _{u \in H_{T}} \varphi(u)=0$ for any $u \in H_{k}$ with $\|u\| \leq \delta$, which implies that any $u \in H_{k}$ with $\|u\| \leq \delta$ are minimum points of $\varphi$. Thus, any $u \in H_{k}$ with $\|u\| \leq \delta$ are $T$-periodic solutions of systems (1.1), and systems (1.1) has infinite $T$-periodic solutions in $H_{T}$. Hence, we complete the proof of our main results. it.

The proof of Theorem 1.4 is almost the same as that in the proof of Theorem 1.3, so we omit

\section{EXAMPLES}

In this section, we will give two examples to illustrate our theorems. 
Example 4.1. Let $F(n+T, x)=F(n, x)$ for any $(n, x) \in\left(\mathbb{Z}, \mathbb{R}^{N}\right)$ and

$$
F(n, x)=\frac{\lambda_{1}}{16}|x|^{2}+\left(\frac{T+1}{2}-n\right)|x|^{7 / 4}+\left(\frac{4}{3} T-n\right)|x|^{3 / 2}, n \in \mathbb{Z} \cap[1, T],
$$

where

$$
F_{1}(n, x)=\left(\frac{T+1}{2}-n\right)|x|^{7 / 4}+(2 T-n)|x|^{3 / 2}, \forall(n, x) \in\left(\mathbb{Z} \cap[1, T], \mathbb{R}^{N}\right)
$$

and

$$
F_{2}(x)=\frac{\lambda_{1}}{16}|x|^{2}-\frac{2}{3} T|x|^{3 / 2}, \forall x \in \mathbb{R}^{N} .
$$

According to (4.26), one has

$$
\begin{aligned}
\left|\nabla F_{1}(n, x)\right| & \leq \frac{7}{8}|T+1-2 n||x|^{3 / 4}+\frac{3}{2}|2 n-T||x|^{1 / 2} \\
& \leq \frac{7}{8}(|T+1-2 n|+\varepsilon)|x|^{3 / 4}+\frac{9 T^{3}}{\varepsilon^{2}}, \forall(n, x) \in \mathbb{Z} \times \mathbb{R}^{N},
\end{aligned}
$$

where $\varepsilon>0$. Then, we obtained that $\left(B_{1}\right)$ holds with $\alpha=3 / 4$ and

$$
f(n)=\frac{7}{8}(|T+1-2 n|+\varepsilon), \quad g(n)=\frac{9 T^{3}}{\varepsilon^{2}} .
$$

According to (4.27), we have

$$
\begin{aligned}
\left|\nabla F_{2}(x)\right| & \leq \frac{\lambda_{1}}{8}|x|+T|x|^{1 / 2} \\
& \leq\left(\frac{\lambda_{1}}{8}+\varepsilon\right)|x|+\frac{T}{\varepsilon}, \forall(n, x) \in \mathbb{Z} \times \mathbb{R}^{N},
\end{aligned}
$$

where $\varepsilon>0$. Then, we obtained that $\left(B_{2}\right)$ holds with

$$
R_{1}=\frac{\lambda_{1}}{8}+\varepsilon, \quad R_{2}=\frac{T}{\varepsilon} .
$$

Now, we verify that $F(n, x)$ satisfies $\left(B_{3}\right)$. In fact,

$$
\begin{aligned}
& \lim _{|x| \rightarrow+\infty} \inf |x|^{-2} \sum_{n=1}^{T} F(n, x) \\
= & \lim _{|x| \rightarrow+\infty} \inf |x|^{-2} \sum_{n=1}^{T}\left[\frac{\lambda_{1}}{16}|x|^{2}+\left(\frac{T+1}{2}-n\right)|x|^{7 / 4}+\left(\frac{4}{3} T-n\right)|x|^{3 / 2}\right] \\
= & \frac{\lambda_{1}}{16} T .
\end{aligned}
$$

It is easy to verify that $M_{3}<\lambda_{1}$. If $T \in\{2,3,4,5,6,7,8,9,10\}$, we can choose $\varepsilon>0$, such that

$$
\lim _{|x| \rightarrow+\infty} \inf |x|^{-2} \sum_{n=1}^{T} F(n, x)=\frac{\lambda_{1}}{16} T>\frac{\left(\frac{\lambda_{1}}{8}+\varepsilon\right)^{2} T}{2\left(\lambda_{1}-\frac{\lambda_{1}}{8}-\varepsilon\right)}=\frac{M_{3}^{2} T}{2\left(\lambda_{1}-M_{3}\right)} .
$$

Thus, the system (1.1) has at least one T-periodic solution by Theorem 1.1.

Example 4.2. Let $F(n+T, x)=F(n, x)$, for any $(n, x) \in \mathbb{Z} \times \mathbb{R}^{N}$ and

$$
F(n, x)=\frac{T-n}{20}|x|^{2}+\frac{4}{7}|x|^{7 / 4}-n|x|^{3 / 2}-|x|+(h(n), x) .
$$


Let

$$
F_{1}(n, x)=\frac{T-n}{20}|x|^{2}-n|x|^{3 / 2}+(h(n), x),
$$

$h: \mathbb{Z} \cap[1, T] \rightarrow \mathbb{R}^{N}, h(n+T)=h(n)$, for $n \in \mathbb{Z} \cap[1, T]$ and

$$
F_{2}(x)=\frac{4}{7}|x|^{7 / 4}-|x| \text {. }
$$

According to (4.33), one has

$$
\begin{aligned}
\left|\nabla F_{2}(n, x)\right| & \leq \frac{T-n}{10}|x|+\frac{3 n}{2}+|h(n)| \\
& \leq\left(\frac{T-n}{10}+\varepsilon\right)|x|+\frac{T^{2}}{\varepsilon}+|h(n)|, \quad \forall(n, x) \in \mathbb{Z} \times \mathbb{R}^{N},
\end{aligned}
$$

where $\varepsilon>0$. Then, we obtained that $\left(B_{4}\right)$ holds with

$$
f(n)=\frac{T-n}{10}+\varepsilon, \quad g(n)=\frac{T^{2}}{\varepsilon}+|h(n)| .
$$

It is easy to verify that $\left(B_{5}\right)$ holds with $\alpha=3 / 4$ and $M_{3}=M_{4}=1$.

Now, we verify that $F(n, x)$ satisfies $\left(B_{6}\right)$. In fact, according to (4.32) and (4.35), we have

$$
\sum_{n=1}^{T} f(n)=\sum_{n=1}^{T}\left(\frac{T-n}{10}+\varepsilon\right)=T\left(\frac{T-1}{20}+\varepsilon\right)
$$

and

$$
\begin{aligned}
& \lim _{|x| \rightarrow \infty} \inf |x|^{-2} \sum_{n=1}^{T} F(n, x) \\
= & \lim _{|x| \rightarrow \infty} \inf |x|^{-2} \sum_{n=1}^{T}\left[\frac{T-n}{20}|x|^{2}+\frac{4}{7}|x|^{7 / 4}-n|x|^{3 / 2}-|x|+(h(n), x)\right] \\
= & \frac{T(T-1)}{40} .
\end{aligned}
$$

When $T \in\{2,3,4\}$, we can choose $\varepsilon>0$, such that

$$
\sum_{n=1}^{T} f(n)=T\left(\frac{T-1}{20}+\varepsilon\right)<\lambda_{1}
$$

and

$$
\begin{aligned}
\lim _{|x| \rightarrow \infty} \inf |x|^{-2} \sum_{n=1}^{T} F(n, x) & =\frac{T(T-1)}{40} \\
& >\frac{1}{2\left(\lambda_{1}-\frac{T-1}{20}-\varepsilon\right)}\left(\frac{T-1}{20}+\varepsilon\right)^{2} \\
& =\frac{1}{2\left(\lambda_{1}-\sum_{n=1}^{T} f(n)\right)}\left(\sum_{n=1}^{T} f(n)\right)^{2} .
\end{aligned}
$$

Thus, the system (1.1) has at least one T-periodic solution by Theorem 1.2.

Acknowledgement. This work was partially supported by the NSF of China (11790271), Guangdong Basic and Applied basic Research Foundation(2020A1515011019), Innovation and Development Project of Guangzhou University. 


\section{REFERENCES}

[1] H. Brezis, L. Nirenberg: Remarks on finding critical points. Commun. Pure Appl. Math. 44 (8-9) (1991), 939-963.

[2] Z. M. Guo, J. S. Yu: Existence of periodic and subharmonic solutions for second-order superlinear difference equations. Sci. China Ser. A 46 (4) (2003), 506-515.

[3] Z. M. Guo, J. S. Yu: The existence of periodic and subharmonic solutions to subquadratic second-order difference equations. J. Lond. Math. Soc. 68 (2) (2003), 419-430.

[4] Z. M. Guo, J. S. Yu.: Periodic and subharmonic solutions for superquadratic discrete Hamiltonian systems. Nonlinear Anal. 55 (7-8) (2003), 969-983.

[5] W. Guan, K. Yang: Existence of periodic solutions for a class of second order discrete Hamiltonian systems. Adv. Difference Equ. 68 (2016).

[6] J. Hu: Existence and Multiplicity of Periodic Solutions for Second-order Discete Hamiltonian Systems. J. Harbin Univ. Sci. Tech. 15 (05) (2010), 119-123.

[7] J. Mawhin, M. Willem: Critical point theory and Hamiltonian systems. Springer-Verlag, New York, 1989.

[8] X. H. Tang, X. Y. Zhang: Periodic solutions for second-order discrete Hamiltonian systems. J. Difference Equ. Appl. 17 (10) (2011), 1413-1430.

[9] Y. F. Xue, C. L. Tang: Existence and Multiplicity of Periodic Solutions for Second-Order Discrete Hamiltonian Systems. J. Southwest China Normal Univ. Nat. Sci. Ed. 31 (1) (2006), 7-12.

[10] Y. F. Xue, C. L. Tang: Multiple periodic solutions for superquadratic second-order discrete Hamiltonian systems. Appl. Math. Comput. 196 (2) (2008), 494-500.

[11] Z. Zhou, J. S. Yu and Z.M. Guo: Periodic solutions of higher-dimensional discrete systems. Proc. Roy. Soc. Edinburgh Sect. A, 134 (5) (2004), 1013-1022.

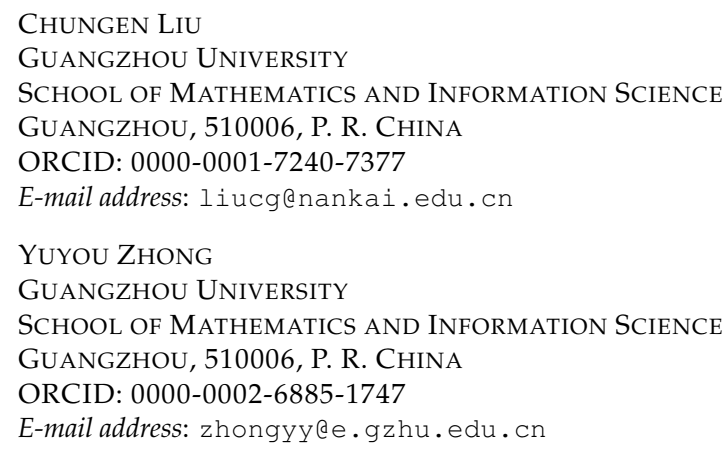

E-mail address: zhongyy@e.gzhu.edu.cn 